\title{
An Approach of Security Risk Evaluation Based on the Bayesian Attack Graph
}

\author{
Wang Hui*, Chen Fuwang and Wang Yunfeng \\ College of Computer Science and Technology, Henan Polytechnic University, Jiaozuo, 454000, P.R. China
}

\begin{abstract}
The evaluation of network risk is a vital task. Nevertheless, there's no approach for looking both the severity of the vulnerabilities and the general status of network security. It can not handle with uncertainty occurred in the process of evaluation. This paper proposes a practical approach named HTV to solve the upper two problems. First, an algorithm using the Bayes Theorem is designed to check the causal dependencies of attack events and their evidence. Then, a model that we call Bayesian Attack Graph (BAG) is proposed to model the attack events and the vulnerabilities and the attack evidence so that the vulnerabilities in system could be identified correctly and availably. Finally, we adapt the definition that we call the degree threat of vulnerability (DVT) to quantify the severity of vulnerabilities. Results in experiments show that this approach can split the vulnerabilities into various levels, so it can help assess the severity of the vulnerabilities and the general status of network security availably.
\end{abstract}

Keywords: Bayesian attack graph, causal dependencies, severity, uncertainty.

\section{INTRODUCTION}

As networks continue to grow both in size and complexity, network security mainly caused by vulnerabilities has become a rising concern. From a security perspective, a strong network is one in which almost all of vulnerabilities can be logically identified and almost all of threats can be comprehensively evaluated. Conversely, current scanning and detecting techniques, such as X-Scan can evaluate threats, but can't comprehensively evaluate them. They can also identify vulnerabilities, but can not availably identify them because of the hardware upgrading and software release. So it is essential to have an availably assessment method to address them.

At present, attack graphs and Bayesian network have been the most popular techniques evaluating network security [1-4]. The attack graph can be applied to identify potential vulnerabilities and comprehensively evaluate network security. In addition, it offers the capability showing all attack paths by analyzing the dependency of vulnerabilities and configurations within networks. However, as it stands in assessment process, lots of uncertainty needs to be dealt with. One of them is the causation of attacks and attack evidence. While for the attack graph, it only shows what is possible without any indication of what is likely [5]. It is often without any way to describe the non-determinism of dependency.

In contrast to the attack graph, Bayesian network has the capability of describing and dealing with uncertainty. And it is an attribute attack graph in essence. Therefore, Bayesian network is more effective than the attack graph for addressing above issues. However, it must achieve the measurement metrics of individual vulnerability.

Currently, one of the standardization of measurement metrics for an individual vulnerability is Common Vulnerability Scoring System (CVSS) [6]. It has the capability of quantifying metrics of vulnerabilities and atomic attacks and providing basic data for assessment of network security. In reality, threat level caused by different vulnerabilities is commonly different considerably in different cost and severity. So we need targeted give priority to dealing with vulnerabilities posing a worst threat to systems based on Bayesian network and CVSS.

\section{LITERATURE REVIEW}

Recent years have seen meaningful effort on the progress of combination of Bayesian network and the CVSS in solving above issues. There are also some methods and models on feasibility of network security assessment. Jone et al. [5] present a method utilizing the combination of Bayesian network and the CVSS to measure probability which vulnerabilities might be exploited successfully. Sawilla et al. [7] consider the importance of graph nodes for attackers to gain privileges of network and apply the Google PageRank algorithm to compute their numeric ranks. Mehta et al. [8] also utilize Google PageRank on different types of attack graphs to evaluate network security. While there still exists drawbacks for posing a worst threat to the system.

To alleviate such drawbacks, Nayot et al. [9] propose a probabilistic model to assess Dynamic Security Risk. By applying logical Bayesian network to analyze vulnerabilities, they model networks to compute probability of multi targets. However, they don't tell us how to give a specific evaluation to overall security of networks. Anoop et al. [10] present a 
method to quantity threats for an individual vulnerability based on attack graphs. They quantitatively input possibility that vulnerabilities can be exploited successfully into its interval and have an effective evaluation to network. But it fails to present an effective way to evaluate the severity of vulnerability. Holm et al. [11] analyze influences that basis data from CVSS have on assessment result and present a quantitative method. However, the quantitative method is so complex that it will be only applied to small networks.

In this paper, we propose a practical method named the hierarchical threat of vulnerability (HTV) to address these limitations. Our major contributions are definitions of Bayesian Attack Graph (BAG) and the degree threat of vulnerability (DTV). We also give a detection algorithm CRDA and a revised algorithm BAGA generating BAG. By defining DVT, our method can be used to divide vulnerabilities in networks into different levels and give an effective assessment to the overall security risk and the severity of vulnerability simultaneously. Compared with [10], the BAGA can be used to generate Bayesian network because it can eliminate cycle paths in attack graphs.

\section{THE CORRELATION BETWEEN ATOMIC AT- TACKS AND ATTACK EVIDENCE}

Within networks, causation of network attacks and attack evidence will be inevitably non-determinate. To correctly account for it, we will define some notions on network security and design a detection algorithm.

Definition 1 Vulnerability: Let $V$ be the set of vulnerabilities and $v_{i} \in V$. For each $v_{i} \in V$, We define $v_{i}$ as a vulnerability in the set $V$.

Definition 2 Atomic Attack: Let $A$ be the set of atomic attacks and $a_{j} \in A$. For each $a_{j} \in A$, We define $a_{j}$ as an atomic attack in the set $A$.

Within the actual network, it is necessary to utilize a series of attack actions exploiting vulnerabilities to gain a single privilege sequentially. So we define an individual action as the atomic attack. Further, it is essentially a set composed by a number of command or operations in a sorted order. That means we can uniformly name the command or operations as elementary operations, represented within the concept as mi. If elementary operations and their built-up sequences are different, as well atomic attacks consisted of them. In Fig. (1), for example, $\left\{\mathrm{m}_{1} \rightarrow \mathrm{m}_{2} \rightarrow \mathrm{m}_{3} \rightarrow \mathrm{m}_{4}\right\}$ and $\left\{\mathrm{m}_{1} \rightarrow \mathrm{m}_{2} \rightarrow \mathrm{m}_{3} \rightarrow \mathrm{m}_{4}\right\}$ are two different atomic attacks.

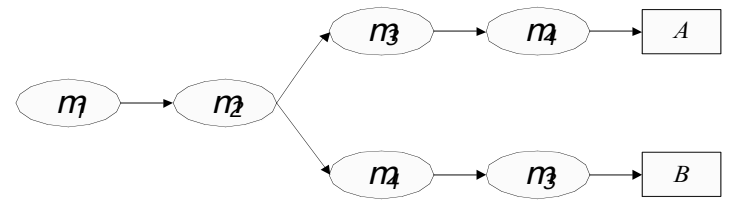

Fig. (1). Atomic attack.

Definition 3 Attack Evidence: Let $O$ be the set of attack evidence and $o_{1} \in O$. For each $o_{1} \in O$, We define $o_{1}$ as one attack evidence in the set $\mathrm{O}$.

In terms of attack evidence, each of them is composed of a series of elementary operations, recorded by monitoring system as the observation data in System log. Because of system malfunction, few of elementary operations must be missing within the System log. Like that, we come to believe that atomic attacks are composed by the same elementary operations, but in different order. That is to say attack evidence from above atomic attacks is always the same. One atomic attack will induce some different attack evidence, but the occurrence of one attack evidence may also depend on multiple atomic attacks. The indeterminacy of their causation is shown in Fig. (2).

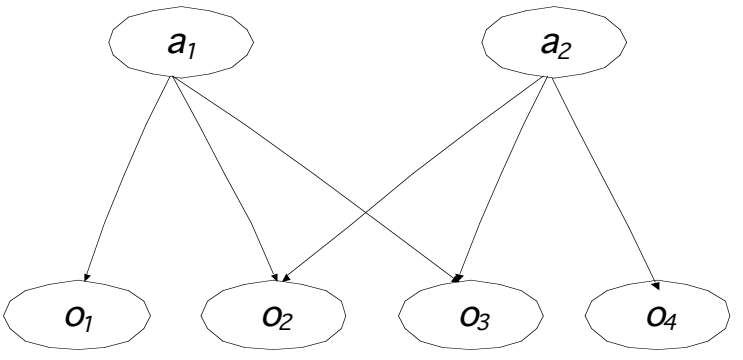

Fig. (2). Causation bayesian network.

In Fig. (2), we can clearly know that $a_{l}$ is likely to induce $o_{1}$ and $o_{2}$ and $o_{3}$ and that $a_{2}$ is likely to induce $o_{1}$ and $o_{2}$. However, the occurrence of $o_{2}$ and $o_{3}$ depends on atomic attacks both $a_{1}$ and $a_{2}$. The causation between $o_{2}$ and $o_{3}$ and $a_{1}$ and $a_{2}$ is their indeterminacy. That's to say we don't know whether $a_{1}$ will induce $o_{2}$ or $o_{3}$, as well $a_{2}$.

To address above indeterminacy, there is a need for an accurate metric measuring possibility that atomic attacks will actually induce attack evidence, namely $P\left(o_{l}, o_{2} \ldots o_{l} \mid\right.$ $\left.a_{1}, a_{2} \ldots a_{j}\right)$. Here, we will employ following notions.

Proposition 1 Here, we assume that the set $X$ consists of a collection of $X_{i}$ and that the set $Y$ consists of a collection of $Y_{m}$. Let the set $Z$ be the subset of $Y$, namely $Z \subseteq Y$. For any integer $r$, with the constraints of $C_{1}(1 \leq r \leq n)$ and $C_{2}$ ( $1 \leq$ $\left.i_{1}<i_{2} \ldots<i_{r}\right)$, if $X_{i 1}, X_{i 2} \ldots X_{i r}$ are independent of each other when the events which the set $Z$ stands for happen, the probability will be computed by the following Eq:

$$
P\left(X_{i 1}, X_{i 2} \ldots X_{i r} \mid Z\right)=\prod_{r=1}^{r} P\left(X_{i r} \mid Z\right)
$$

Let $O=\left\{o_{1}, o_{2} \ldots o_{l}\right\}$ and $A=\left\{a_{1}, a_{2} \ldots a_{j}\right\}$ be the nodesets of Bayesian network $\zeta$. We assume $O$ is independent when events which the set $A$ stands for happen. Thus we will logically obtain Eq. (1) based on Proposition 1.

$$
P\left(o_{1}, o_{2} \ldots o_{l} \mid a_{1}, a_{2} \ldots a_{j}\right)=\prod_{l=1}^{l} P\left(o_{l} \mid a_{1}, a_{2} \ldots a_{j}\right)
$$

More unconditionally, for any attack evidence $o_{l} \in O$, if $A=\left\{A_{l}, A_{2} \ldots A_{l}\right\}$ is a different form of the set $A$, which $A_{l}$ is also one subset of $A$, and the set $A_{l}$ keeps attack evidence $o_{l}$ and all subsets within $\left\{A_{1}, A_{2} \ldots A_{l-1}\right\}$ mutual independence, then $P\left(o_{1}, o_{2} \ldots o_{l} \mid a_{1}, a_{2} \ldots a_{j}\right)$ can be further computed from Eq. (1), using the multiply:

$$
\begin{aligned}
P\left(o_{1}, o_{2} \ldots o_{l} \mid a_{1}, a_{2} \ldots a_{j}\right) & =\prod_{l=1}^{l} P\left(o_{l} \mid a_{1}, a_{2} \ldots a_{j}\right) \\
= & P\left(o_{l} \mid A l\right)
\end{aligned}
$$


However, as is usually the condition of Eq. (2), it isn't easy to compute the value of $P\left(o_{l} \mid A_{l}\right)$, since only the independence of both two is really complex. Thus we will define the proportion of $n_{l}$ (the number of elementary operations within $o_{l}$ ) and $k_{s}$ (the number of elementary operations within $a_{j}$ equals to $\left.n_{l}\right)$ to represent $P\left(o_{l} \mid A_{l}\right)$. Here, we assume that the set of $k_{s}$ corresponding to $\left\{A_{1}, A_{2} \ldots A_{l}\right\}$ is $\left\{k_{1} k_{2} \ldots k_{s}\right\}$, then we obtain Eq. (3).

$$
P\left(o_{l} \mid A_{l}\right)=\prod_{s=1}^{s} \frac{n_{l}}{k_{s}}
$$

Defining the probability $P\left(o_{1}, o_{2} \ldots o_{l} \mid a_{l}, a_{2} \ldots a_{j}\right)$ as Eq. (4):

$$
P\left(o_{1}, o_{2} \ldots o_{l} \mid a_{1}, a_{2} \ldots a_{j}\right)=\prod_{l=1}^{l} \prod_{s=1}^{s} \frac{n_{l}}{k_{s}}
$$

In Fig. (2), we assume that elementary operations of $a_{1}, a_{2}, o_{1}$ and $o_{2}$ as follows: $a_{1}=\left\{m_{1}, m_{2}, m_{3}, m_{4}\right\}$ and $a_{2}=$ $\left\{m_{5}, m_{6}, m_{7}, m_{8}\right\}, \quad o_{1}=\left\{m_{1}, m_{2}, m_{4}\right\}, o_{2}=\left\{m_{1}, m_{2}, m_{7}\right\}$. From Eq.(4), we finally know that $P\left(o_{1} \mid a_{1}\right)=1, P\left(o_{1} \mid a_{2}\right)=0, \quad P\left(o_{2} \mid a_{1}\right)=2 / 3$, $P\left(o_{2} \mid a_{2}\right)=1 / 3$.

Definition 4 For atomic attacks and attack evidence, the vector $\delta_{l}=\left(\alpha_{l l}, \alpha_{l 2}, \ldots \alpha_{l j}\right)$ is employed to describe their causation. In it, $\alpha_{l j}$ actually represents the causation between $a_{j}$ and $o_{l}$, and $\operatorname{RDS}\left(\alpha_{l j}\right)$ eventually conveys the result of $\alpha_{l j}$. If there exists the causation of two, then the value of $\operatorname{RDS}\left(\alpha_{l j}\right)$ will equal to 1 . Else it equals to 0 . Further more, we utilize $\phi_{l j}=\left(\lambda_{l l}, \lambda_{l 2}, \ldots \lambda_{l j}\right)$ to describe the posterior probability of atomic attacks corresponding to $\delta_{l}$, and we use $\lambda_{l j}$ to convey above posterior probability. By Eq. (3), we know $P\left(o_{l} \mid a_{j}\right)=n / s(n, s$, the number of elementary operations corresponding to $o_{l}$ ). So, the Eq. (5) is as follows based on the Bayes' theorem:

$$
\begin{aligned}
l_{l j}=p\left(a_{j} \mid o l\right)= & \frac{p\left(a_{j}\right) \cdot p\left(o l \mid a_{j}\right)}{p(o l)} \\
& =\frac{p\left(a_{j}\right)}{p(o l)} \cdot \frac{k_{S}}{n_{l}}
\end{aligned}
$$

To calculate $\lambda_{l j}$, we must know the prior probability of $a_{j}$ and $P\left(o_{\nu}\right)$. In fact, it has already happened when we observed $o_{l}$ from monitoring system. So $P\left(o_{l}\right)=1$. In this case, if the value of $\max (\phi)$ equals to $\lambda_{l m}$, there will be the result that $a_{m}$ produces $o_{l}$. Here we give Algorithm 1 (causal relation detection algorithm, CRDA) to determine their causation.
The uppermost essence of procedure determining the causation is described as follows: During the inner loop FOR....DO (05-10), it begins to calculate the posterior probability of $a_{j}$ which there exists causation between $o_{l}$ and $a_{j}$; then the maximum value among above posterior probability is taken for the result of detection causation (11); in 13 line, the causation denoted by $R\left(o_{l}-a_{j}\right)$ is pushed into $\Psi$. By running Algorithm 1, we will obtain the atomic attacks from $\Psi$ based on observed attack evidence.

\section{MODELING ATTACK MODEL WITH BAYESIAN NETWORK AND CVSS}

To efficiently and synthetically identify potential vulnerabilities, we built the system architecture of attack model and model $B A G$ by analyzing transitions of system states and improved an algorithm generating attack graphs.

\subsection{System Architecture of Attack Model}

The result carrying out an atomic attack successfully is one transition of system states. So we keep the process exploiting vulnerabilities regard as transitions of system states. Under the prerequisite of network attacks, only if attackers carry out attack action and obtain privileges, can system states be transited. And there will be attack evidence with it. Hence, Let STS=(precondition, $a_{j}$, postcondition, $v_{i}, o_{l}$ ) be transitions of system states. In STS, the precondition denotes preconditions of atomic attacks. And it consists of permissions for source host dst_access and destination host. The $s r$ _ $d s t$ is the connection of source host and destination host. The postcondition denotes results of states transitions, and it means the ascension of attackers' ability. In this section, the ability will be represented by attackers' privileges which includes None, User, Root. Fig. (3) is the model of System State Transition.

The process attacking networks commonly includes a series of complex changes of system states carried out in the order loop of staging attacks and accessing to the host privileges consecutively. And it will not stop until the attacker access to the most important privileges. During it, there will be simultaneously attack evidence. So we can build the network model based on observed attack evidence and the primary steps are as follows:

First, it begins a collection of attack evidence, atomic attacks and vulnerabilities existing in networks and establishes

\begin{tabular}{|c|c|c|}
\hline $\begin{array}{l}\text { Input: } \\
\text { Output: }\end{array}$ & $\begin{array}{l}\Theta \ldots . . . \text { Causation Bayesian Network } \\
\Psi \text {....-the set of causation } \mathbf{a}_{\mid j}\end{array}$ & \\
\hline Algorithm & $01 \psi \leftarrow \varnothing$ & O8 END IF \\
\hline & 02 FOR l $=1$ TOK & $09 \quad \mathrm{M}=\mathrm{M} \cup\left\{\lambda_{i j}\right\}$ \\
\hline & $03 \quad \delta_{1}=\left(\alpha_{111}, \alpha_{12}, \ldots \alpha_{1 j}\right)$ & 10 END FOR \\
\hline & $04 \quad \mathrm{M}_{1} \leftarrow \varnothing$ & $11 \quad \max (M)=\lambda_{i}$ \\
\hline & $05 \quad$ FOR $\mathrm{j}=1 \mathrm{TOm}$ & 12 END FOR \\
\hline & 06 IF RD S $\left(f_{j}=1\right)$ THEN & $13 \Psi=\Psi\{\mathrm{R}(\mathrm{ol}$ aj $)\}$ \\
\hline & $07 \lambda_{j}=\left[p\left(a_{i}\right) / p\left(o_{l}\right)\right] \cdot\left[n / k_{s}\right]$ & 14 RETURN $\Psi$ \\
\hline
\end{tabular}
the corresponding lists of them. Second, it must find out all possible atomic attacks yet obtained privileges by attackers

Algorithm 1. Causal relation detection algorithm ( $\theta)$. 


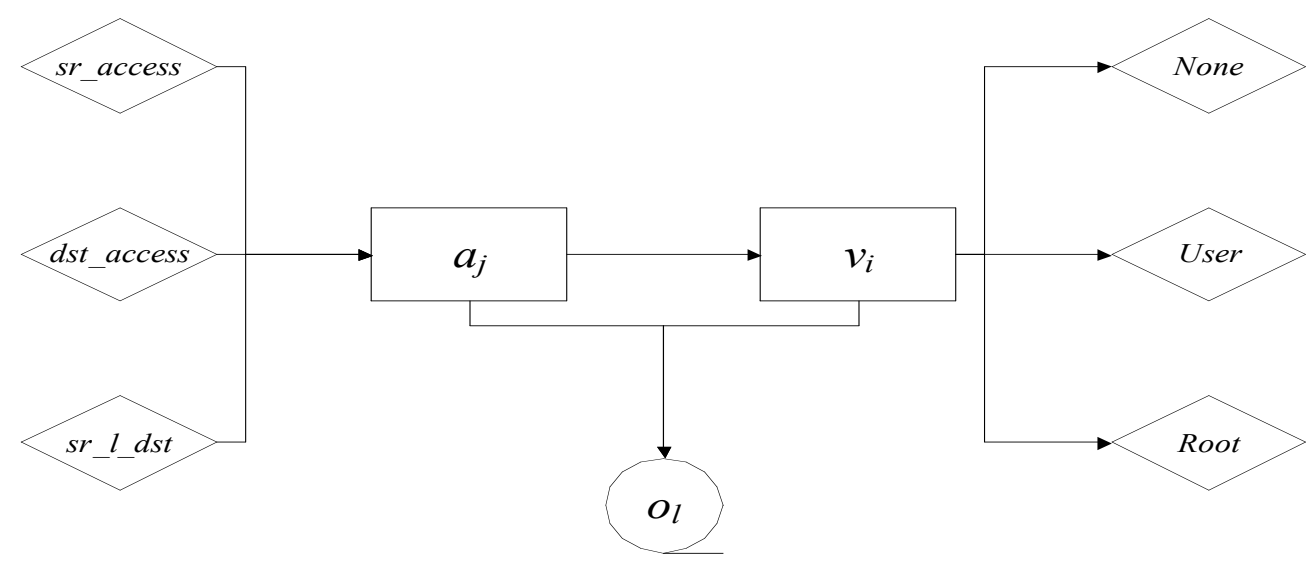

Fig. (3). System state transition.

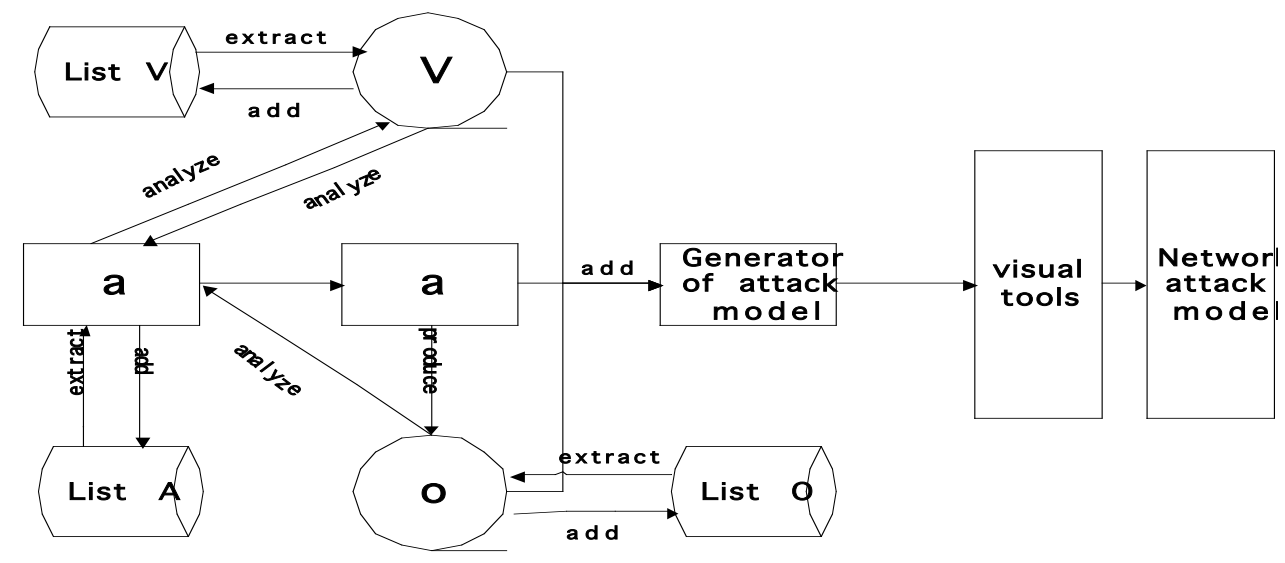

Fig. (4). System architecture of attack model.

and attack evidence so that we can identify vulnerabilities as much as possible by analyzing vulnerabilities. Finally, it should be added to the attack model. The attack model is as Fig. (4).

\subsection{Bayesian Attack Graph}

Definition 5 Bayesian Attack Graph A Bayesian Attack Graph is a tuple $B A G=(T, W, \Pi, E, C)$, where

(1) $T=V \cup A \cup O$. $\mathrm{T}$ is the set of different types of nodes consisted of $B A G$. $V=V_{0} \cup V_{d}$. $V_{0}$ denotes the set of initial states of nodes and $V_{d}$ denotes the set of accessible states of nodes within $B A G$. For any $t \in V \cup A$, either the value of it is True or False. Here, we let Pre(t) be the parent set of node $\mathrm{t}$ and $\operatorname{Post}(t)$ be the subset of node $t$.

(2) $\mathrm{W}$ is the probability of $o_{l}$ which is produced by $a_{j}$. For $\forall w \in W$, it's a tuple of $(n, m)$. n denotes the number of elementary operations in $o_{l}$ which is observed by monitoring system and $m$ is the number of elementary operations within $a_{j}$ equals to $\mathrm{n}$ corresponding to $o_{l}$. In Fig. (2), for example, $n\left(o_{2}\right)=3$ and $m\left(a_{1}, o_{2}\right)=2$, so the value of $w\left(a_{1}, o_{2}\right)$ equals to $m\left(a_{1}, o_{2}\right) / n\left(o_{2}\right)=2 / 3$.

(3) $\Pi(B A G) \subset\{P(t) \cup P(t \mid \operatorname{Pre}(t))\}$, is the set of probability distribution on nodes within $B A G$.

(4) $E \subset\left(\left[\left(V_{0} \cup V_{d}\right) \times A\right] \cup\left[A \times\left(V_{0} \cup V_{d}\right)\right] \cup[A \times O]\right)$, denotes the set of directed edges associated with nodes in $B A G$.
(5) $C$ denotes the constraints that must be satisfied for the $B A G$, where

a) For any node $t \in A$, their parent nodes represent within $B A G$ as logical OR-nodes and satisfy with the constraint $\left(\operatorname{Pre}(t) \subset V_{0}\right) \wedge\left(\operatorname{Post}(t) \subset V_{d}\right)$

b) For any node $t \in V$, their parent nodes represent within $B A G$ as logical $A N D$-nodes and satisfy with the constraint $(\exists t \subset A) \vee(\operatorname{Post}(t) \subset A)$;

c) For any node $t \in T$, it always satisfies with the normative constraint of probability measure, namely $\Pi(B A G) \subset\{P(t) \cup P(t \mid \operatorname{Pre}(t)) \rightarrow[0,1]$.

Algorithm 2 is used to generate Bayesian Attack Graph automatically. Its' procedure is described as follows: First, it provides a process initializing all nodes and parameters within $B A G(1-3)$. The $1^{\text {st }}$ line sets up a list of privileges by calling the MARKPLACE process and gives a blank state to $B A G$. It also gives the attackers access to Root. In line $2 \sim 3$, it creates a queue in network state and adds the init_state to the queue to initialize the $B A G$. Pseudocodes4-16 is the second stage with loops. The $4^{\text {th }}$ line provides an outer loop to extract nodes from init_state. Pseudocodes5-13 is an inner loop. For any nodes in the init_state, we traverse all atomic attacks associated with it in the $A$ list (5). If the atomic attack attacks the node according to $\operatorname{CVEDB}(6)$, it will find out the attack evidence produced by it from the $O_{-} \operatorname{list}(7 \sim 8)$ and 
Algorithm 2. Bayesian attack graph generation algorithm BAGA.

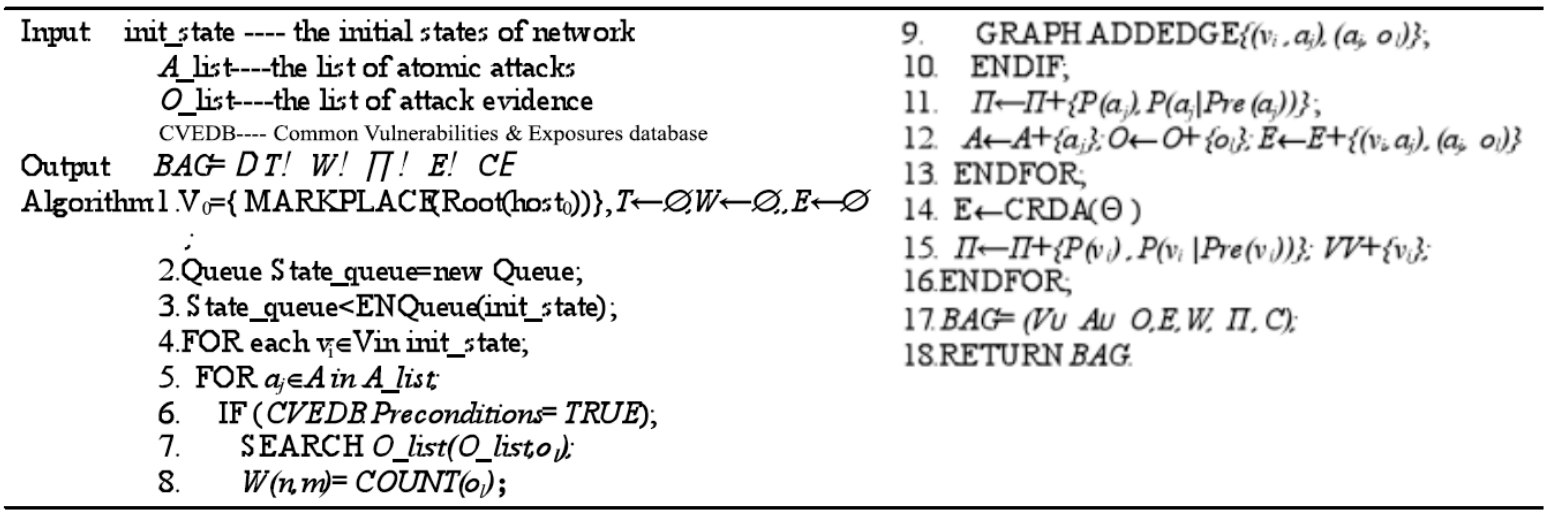

make them the node $T(11 \sim 12)$ and their edges $E(9)$. The loop will continue until the init state is null. Finally, it will add above nodes $T$ and edges $E$ to $B A G$ and return the $B A G$ eventually (17 18).

According to the real-time observed attack evidence, Algorithm 2 can automatically generate Bayesian network consisted of nodes of atomic attacks, attack evidence and vulnerabilities and show vulnerabilities existing in networks, which makes it effectively identify potential vulnerabilities.

When generating $B A G$ by running Algorithm $\mathbf{2}$, there will be two issues: 1) Privileges, such as User, are the fallout exploiting vulnerabilities, but will not be a type of nodes in its own right; 2) There are relationships of one-to-one correspondence among vulnerabilities, atomic attacks and attack evidence each other, but it can't display the relationship what reflects their causation in $B A G$. So, we make up rules alleviating above drawbacks.

(1) We name nodes of vulnerabilities as "vulnerability number $\left(v_{i}\right)$ ". For example, CVE575( $\left.v_{l}\right)$ means the vulnerability $v_{l}$ within networks is Buffer Overflow.

(2) We name privilege as "access level(Host)". For example, $\mathrm{R}(\mathrm{H} 0)$ means that attackers have obtained the Root privilege from Host 0 .

(3) We name nodes of privileges as "access level(Host) $a_{j} \_$vulnerability $\left(v_{i}\right)$ ". For example, $R(H 0) \_a_{2}$ CVE575 $\left(v_{l}\right)$ means attackers will attack $v_{l}$ using $a_{2}$ when he has obtained the Root privilege from Host0 by exploiting Buffer Overflow Vulnerability.

(4) We name nodes of attack evidence as " $a_{i_{-}} v_{l_{-}} o_{l}$ ”. For example, $a_{2} v_{1} o_{1}$ means that it will produce $o_{l}$ when Buffer Overflow $\left(v_{l}\right)$ within networks is exploited by $a_{2}$.

\section{ASSESSMENT OF THREAT WITH BAG}

We must targeted give priority to dealing with vulnerabilities posing a worst threat to systems considerably in different cost and severity. Here, we define DVT and formulas to quantifying the severity of vulnerabilities.

\subsection{Probability of nodes in BAG with CVSS}

Definition 6 The degree of vulnerability threat (DVT) is a function mapping each vulnerability in $B A G$ to probability interval $[0,1]$, described mathematically $p: V \rightarrow[0,1]$. It can be used to measure the level of privilege attackers will obtain by exploiting vulnerabilities. The larger of its value, the higher of the level of privilege is and the more severe of vulnerabilities are.

The $B A G$ could be effectually utilized to assess the severity of vulnerabilities within networks. To accurately measure DVT, it is prioritized and necessary to assign self-probability of atomic attack node $s\left(a_{j}\right)$ and vulnerability node $s\left(v_{i}\right)$, which their self-probability are from the attribute "AccessComplexity" of CVSS. For any vulnerability, the value of its self-probability is commonly steadfast. So we make the rule that $s\left(v_{i}\right)$ equals to 1.0 . The values of $s\left(a_{j}\right)$ in our network are as Table 1.

Table 1. Basic score of CVSS.

\begin{tabular}{|c|c|c|}
\hline \multirow{2}{*}{ Attribute of CVSS } & Level & $\mathbf{S}\left(\mathbf{a}_{\mathbf{j}}\right)$ \\
\hline \hline \multirow{3}{*}{ Access Complexity } & Low & 0.71 \\
\cline { 2 - 3 } & Medium & 0.61 \\
\cline { 2 - 3 } & High & 0.35 \\
\hline
\end{tabular}

\subsection{Hierarchical threats of vulnerabilities}

More generally, the probability of nodes in Bayesian network associate with the attribute of its parents and itself. In this section, for any node $t$ within Bayesian network, we assume that the number of nodes that are independent of each other is $K$ in the set pre(t) and the array $d[i](1 \leq i \leq k)$ refers to the node number. Based on Bayes' theorem:

$$
P(t)=(t \mid p r(t))=p(t \mid p r(t), p r(t) \rightarrow t)
$$

Considering the logical relation $d_{t}$ among its parents, the probability of node $t$ is defined as Eq. (7):

$$
p(t)= \begin{cases}P\left(\bigcap_{i=1}^{k} d[i]\right)=\prod_{i=1}^{k} P(d[i]) \quad d t=A N D \\ p\left(\bigcup_{i=1}^{k} d[i]\right)=\prod_{i=1}^{k}\{1-P(d[i])\} \quad d t=O R\end{cases}
$$

Definition 7 For any $t \in T(B A G)$ within $B A G$, the absolute probability $p(t)$ is defined as follows: 


$$
P(t)=\left\{\begin{array}{cl}
1.0 & \forall t \in \text { root; } \\
\frac{s(t) \cdot \prod_{i=1}^{k} p[d(i)] \cdot p\left(o_{l} \mid t\right)}{p\left(o_{l}\right)} & \forall t \in A ; \\
s(t) \cdot\left[1-\prod_{i=1}^{k}(1-p(d(i)))\right] & \forall t \in V_{0} \cup V_{d} A N D \\
& \forall t \in \text { root }
\end{array}\right.
$$

To accurately calculate DVT considerately in attack evidence, we discuss the probability distribution of 3 kinds of nodes. (1) root nodes. Root nodes represent one kind of nodes that have been successfully exploited in the beginning state of networks. So the actual probability $p(t)=1.0$.(2) $\forall t \in A$. The probability of atomic attacks associates with its parents, attacks evidence and itself. So the ac-

tual probability is $p(t)=s(t) \cdot \prod_{i=1}^{k} p[d(i)] \cdot p(o l \mid t) / p(o l)$.

(3) $\forall t \in V_{0} \cup V_{d} A N D \forall t \notin$ root. The probability of vulnerabilities exploited successfully associates with its parents, and itself. So the actual probability is

$$
s(t) \cdot\left[1-\prod_{i=1}^{k}(1-p(d(i)))\right] .
$$

Because of the risk and crime cost, only the path maximizing the success rate exploiting vulnerabilities may be selected to reach destination nodes by attackers eventually. Hence, we define the function of DVT based on Definition4 and Definition6 as Definition8:

Definition 8 Given $B A G=(T, W, \Pi, E, C)$, for any node $t \in V_{0} \cup V_{d}, \quad p_{\max }$ represents its DVT and $p_{\max }=s(t) \cdot M A X$ $\left\{\frac{P[d(i)]}{p(t)} \mid d(i)\right.$ is a parent of node $t$ and $\left.i=1,2,3, \cdots k\right\}$.

In fact, $p[d(i)] / p(t)$ is the largest success rate exploiting vulnerabilities among all nodes of $p[d(i)]$. So $p_{\max }$ can be used to measure the most possible threats of vulnerabilities exploited successfully.

\section{IMPLEMENTATION AND EXPERIMENTAL RESULTS}

\subsection{Implementation and Network Configuration}

In this section, the validity of our method is verified by a simulation. Fig. (5) shows a sample network, which will be used to demonstrate the implementation of our method.

Network Configuration: Experimental network is separated internal network and external network by Firewall1. The internal network includes the secured area of M1, M2,

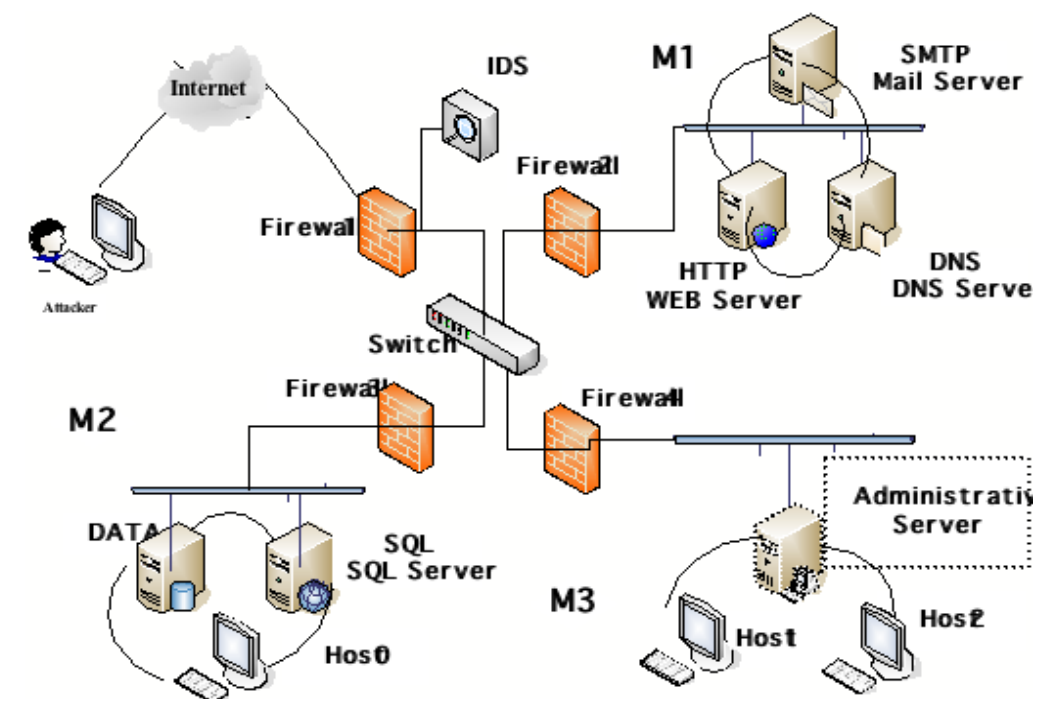

Fig. (5). Example experimental network.

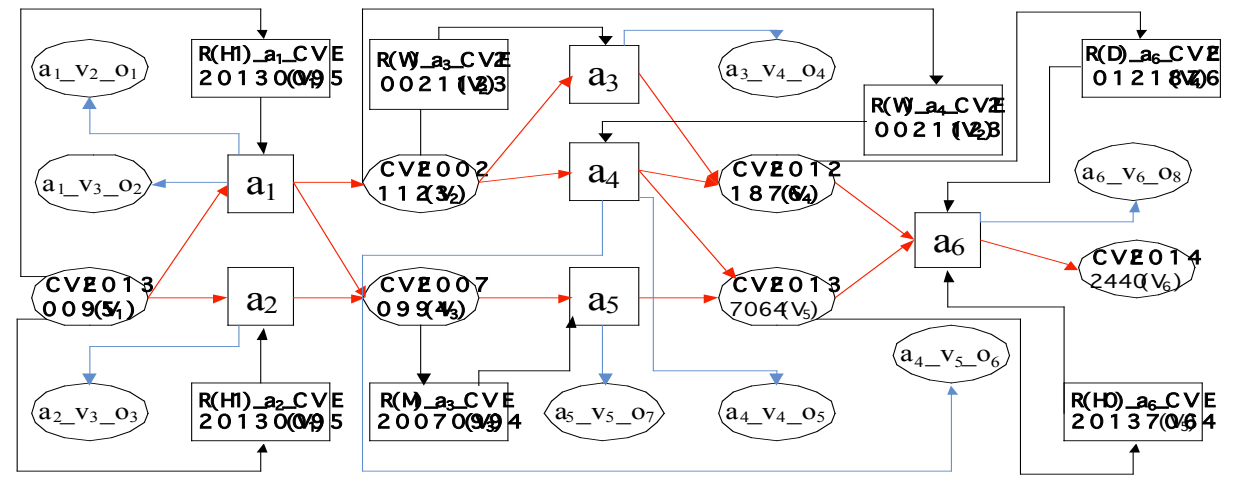

Fig. (6). The bayesian attack graph. 
Table 2. Vulnerability information of host.

\begin{tabular}{|c|c|c|c|c|c|c|c|}
\hline $\begin{array}{l}\text { Secured } \\
\text { Area }\end{array}$ & Node & Host & Vulnerability & Service & CVE & $\begin{array}{l}\text { Type of Privi- } \\
\text { lege }\end{array}$ & $\begin{array}{l}\text { Score of Access } \\
\text { Conexity }\end{array}$ \\
\hline MB & $\mathrm{V}_{1}$ & $\begin{array}{l}\text { Host } 1 / \\
\text { Host } 2\end{array}$ & $\begin{array}{l}\text { Unintended Content Loading } \\
\text { Vulnerability }\end{array}$ & $\begin{array}{c}\text { RSH/ } \\
\text { HTTP/SQL }\end{array}$ & $\begin{array}{l}\text { CVE-2013- } \\
0095\end{array}$ & Non/User & 0.71 \\
\hline \multirow{3}{*}{ M1 } & $\mathrm{V}_{2}$ & $\begin{array}{l}\text { Web } \\
\text { Server }\end{array}$ & $\begin{array}{l}\text { Buffer overflow in the } \\
\text { authentication function }\end{array}$ & НTTP & $\begin{array}{l}\text { CVE-2002- } \\
1123\end{array}$ & User & 0.71 \\
\hline & $\mathrm{V}_{3}$ & $\begin{array}{l}\text { Mail } \\
\text { Server }\end{array}$ & $\begin{array}{c}\text { Remote code execution in } \\
\text { SMTP }\end{array}$ & SMTP & $\begin{array}{l}\text { CVE-2007- } \\
0994\end{array}$ & User/Root & 0.61 \\
\hline & $\mathrm{V}_{4}$ & $\begin{array}{l}\text { DNS } \\
\text { Server }\end{array}$ & $\begin{array}{l}\text { Col Element Remote Code } \\
\text { Execution Vulnerability }\end{array}$ & DNS & $\begin{array}{l}\text { CVE-2012- } \\
1876\end{array}$ & Non/User/Root & 0.61 \\
\hline \multirow{2}{*}{ M2 } & $\mathrm{V}_{5}$ & $\begin{array}{l}\text { Host } 0 / \\
\text { DATA }\end{array}$ & $\begin{array}{c}\text { Cross-site scripting (XSS) } \\
\text { Vulnerability }\end{array}$ & HHS/FTP & $\begin{array}{l}\text { CVE-2013- } \\
7064\end{array}$ & Root & 0.35 \\
\hline & $\mathrm{V}_{6}$ & $\begin{array}{c}\text { SQL } \\
\text { Server }\end{array}$ & $\begin{array}{l}\text { Unspecified vulnerability in the } \\
\text { MySQ }\end{array}$ & SQL & $\begin{array}{l}\text { CVE-2014- } \\
2440\end{array}$ & User/Root & 0.35 \\
\hline
\end{tabular}

M3, which are separated by firewall each other. The external network includes the Internet and several hosts which provide services as Fig. (5), and the attack host 0 is the destination host.

\subsection{Experimental Results}

Vulnerabilities within networks will enable an attacker to gain control of hosts. In this section, the vulnerabilities and servers within the sample network are shown in Table $\mathbf{2}$ and the $B A G$ generated by running Algorithm 2 is shown in Fig. (6).

With the growing of intrusion for networks, the ability of attackers obtaining higher permissions is continuously improved. Within networks, the IDS deployed on the mirror port can detect potential intrusion behavior and analysis host permissions gained by attackers by collecting and analyzing various network data. To hierarchically assess the severity of vulnerabilities, we will introduce several parameters in the experimental process as Table 3 .

Table 3. Parameters of running experiment.

\begin{tabular}{|c|c|c|c|c|}
\hline Test & $\boldsymbol{N}$ & $\boldsymbol{q}_{\boldsymbol{R}}$ & $\boldsymbol{q}_{U}$ & $\boldsymbol{q}_{\boldsymbol{N}}$ \\
\hline \hline 1 & 100 & 0.4 & 0.4 & 0.2 \\
\hline 2 & 200 & 0.5 & 0.3 & 0.2 \\
\hline 3 & 300 & 0.6 & 0.3 & 0.1 \\
\hline
\end{tabular}

The number of atomic attacks $N$ : It represents the number of intrusion behavior which has recorded by the IDS in an experiment.

Attack rate q: It represents the percentage of atomic attacks used to obtain a level of privilege. It will include three kinds of attack rate: Root rate (disproportionately, set $q_{R}$ ), User rate (disproportionately, set $q_{u}$ ) and None rate (disproportionately, set $q_{N}$ ). Root rate means that the percentage of atomic attacks used to obtain Root privilege is $q_{R}$ and $q_{R}=n($ Root $) /[n($ Root $)+n($ User $)+n$ (None $\left.)\right]$. Like that, $q_{u}=n($ User $) /[n($ Root $)+n($ User $)+n$ (None) $]$ and $q_{N}$ $=n($ None $) /[n($ Root $)+n($ User $)+n$ (None $)]$. Considering the relation of Attack rate and the level of privilege, we will as- sume that only if the value of $p_{\max }\left(v_{i}\right)$ is between $q_{U}+q_{N}$ and $q_{U}+q_{N+} q_{R}$ (intervally, $p_{\max }\left(v_{i}\right) \in\left[q_{U}+q_{N}, q_{U}+q_{N+} q_{R}\right]$ ), can attackers enable to obtain the Root privilege. And likewise, we know $p_{\max }\left(v_{i}\right) \in\left[q_{U}+q_{N}, q_{N}\right]$ for User privilege and $p_{\max }\left(v_{i}\right) \in[$ $\left.q_{N}, 0\right]$ for None privilege.

Fig. (7) graphically shows DVT within networks. The blue line represents DVT. The minimum of Root privilege is represented by $q_{R}$ with purple line and the ceiling is 1.0 . The minimum of User privilege is represented by $q_{N}$ with red line and the ceiling is represented by $q_{U}$ with black line. The ceiling of None privilege is represented by $q_{N}$ and the minimum is 0 . For example, if $p_{\max }\left(v_{i}\right) \in\left[q_{R}, 1.0\right]$, we think that attackers will obtain Root privilege by exploiting $v_{i}$.

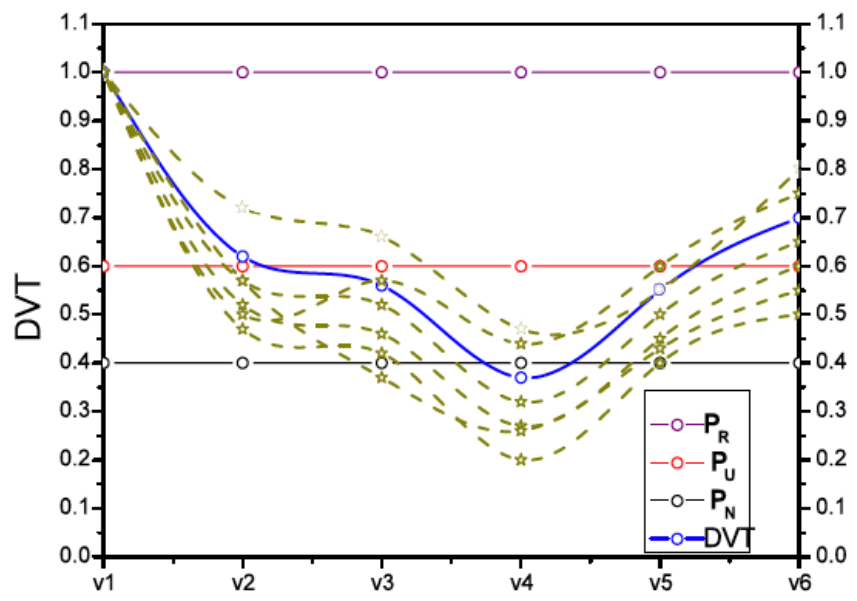

Fig. (7). The severity of vulnerabilities.

In Fig. (7), vulnerabilities are hierarchically divided into 3 groups. (1) $v_{1}, v_{2}, v_{6}$. Their values of DVT are in the interval $[0.6,1.0]$ and they will be exploited by attackers to obtain Root privilege in networks. (2) $v_{3}, v_{5}$. Their values of DVT are in the interval $[0.4,0.6]$ and they will be exploited by attackers to obtain User privilege in networks. (3) $v_{4}$. Its value of DVT is in the interval $[0,0.4]$ and attackers will not obtain privilege by exploiting it in networks. In crosswise comparison, if we sort by severity of vulnerabilities, the order of $v_{1}, v_{2}, v_{6}$ should be $v_{1}, v_{6}, v_{2}$. And likewise, the order of 
$v_{3}, v_{5}$ should be $v_{3}, v_{5}$. Further more, in longitudinal comparison, if we sort by severity of vulnerabilities, the order of them should be $v_{1}, v_{6}, v_{2}, v_{3}, v_{5}, v_{4}$. So we can draw a conclusion from Fig. (7) that the priority to dealing with vulnerabilities is $v_{1}, v_{6}, v_{2}, v_{3}, v_{5}, v_{4}$.

Fig. (8) graphically shows the result of assessment to the overall security of networks. There are overall three lines: black line represents the threshold threatening to destination host; red line A represents the trend that the DVT of $v_{6}$ has with the growing of atomic attacks when Host 0 is taken for the destination host; blue line B represents the trend that the DVT of $v_{6}$ has with the growing of atomic attacks when Host 0 is not taken for the destination host.

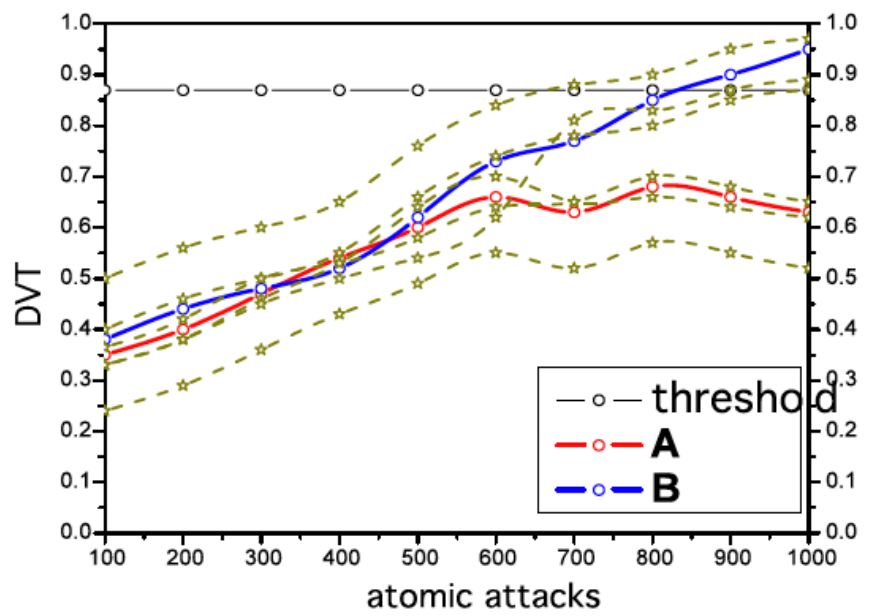

Fig. (8). The overall security of target network.

The line A shows that the DVT of $v_{6}$ first becomes larger and then tends to stability. It demonstrates that for the nondestination host, attackers don't try to obtain privilege with higher level. So, the trend of overall security of networks in the situation will be the same as the line A. The B shows that the DVT of $v_{6}$ has been growing until it is more than threshold. It suggests that the attacker will try to obtain the highest authority of destination host. Therefore, our method can support the network early warning for managers so that they will make a clear decision.

\section{CONCLUSION}

The paper proposes a method to evaluate overall security risk of networks and severity of vulnerabilities simultaneously by using Bayesian network and CVSS. The method is practical in the assessment of them, as well as determination of the causation between atomic attacks and attack evidence. By running it, we can give the different priority to dealing with vulnerabilities and support the network early warning for managers so that they will make a clear decision.
For future work, we will further study on the uncertainty within networks and verify the effectiveness of our method in more complex networks.

\section{CONFLICT OF INTEREST}

The authors confirm that this article content has no conflict of interest.

\section{ACKNOWLEDGEMENTS}

This work was financially supported by the National Natural Science Foundation of China (No. 61300216), supported by Research Fund for the Doctoral Program of Higher Education of China (No. 20124116120004), and supported by Educational Commission of Henan Province of China (No. 13A510325).

\section{REFERENCES}

[1] B. Mahmoud, N. Larrieu, and A. Pirovano, "A Risk Propagation Based Quantitative Assessment Methodology for Network Security Aeronautical Network Case Study", In: Proceedings of the Integrated Communications, Navigation and (ICNS), 2011, pp. 1-9.

[2] M. Frigault, and L.Y. Wang, "Measuring network security using Bayesian network-based attack graphs", In: Proceedings of the $3^{\text {rd }}$ IEEE International workshop on Security, Trust, and Privacy for Software Applications, 2008, pp. 698-703.

[3] A.M. Xie, W.P. Wen, L. Zhang, J.B. Hu, and Z. Chen, “Applying Attack Graphs to Network Security Metric", In: Proceedings of the Multimedia Information Networking and Security (MINES), 2009, pp. 427-431.

[4] M.Salim, E. Al-Shaer, and L. Khan, "A novel quantitative approach for measuring network Security”, In: Proceedings of the $27^{\text {th }}$ Conference on Computer Communications (INFOCOM), 2008, pp. 1957-1965.

[5] J. Homer, X.M Ou, and D. Schmidt, "A Sound and Practical Approach to Quantifying Security Risk in Enterprise Networks", Kansas State University Technical Report, 2009, pp 1-15.

[6] P. Mell, K. Scarfone, and S. Romanosky, "Common Vulnerability Scoring System”, Security \& Privacy, IEEE, vol. 4, no. 6, pp. 85$89,2006$.

[7] R. Sawilla, and X.M. Ou, "Identifying critical attack assets in dependency attack graphs", In: Proceedings of the $13^{\text {th }}$ European Symposium on Research in Computer Security, 2008, pp. 18-34.

[8] V. Mehta, C. Bartzis, H. Zhu, E. Clarke, and J. Wing, "Ranking attack graphs", In: Proceedings of Recent Advances in Intrusion Detection, 2006, pp. 127-144.

[9] P. Nayot, D. Rinku, and R. Indrajit, "Dynamic Security Risk Management Using Bayesian Attack Graphs", Dependable and Secure Computing, IEEE Transactions, vol. 34, no. 1, pp. 61-74, 2011.

[10] A. Singhal, and X.M. Ou, "Security Risk Analysis of Enterprise Networks Using Attack Graphs", SpringerBriefs in Computer Science, Springer, New York, 2011, pp. 13-23.

[11] H. Holm, M. Ekstedt, and D. Andersson, "Empirical Analysis of System-Level Vulnerability Metrics through Actual Attacks", Dependable and Secure Computing, IEEE Transactions, vol. 9, no. 6, pp. 825-837, 2012.

Received: June 10, 2015

(C) Hui et al.; Licensee Bentham Open.

This is an open access article licensed under the terms of the (https:/creativecommons.org/licenses/by/4.0/legalcode), which permits unrestricted, noncommercial use, distribution and reproduction in any medium, provided the work is properly cited. 